\title{
Detectability of Planets in Wide Binaries by Ground-Based Relative Astrometry with AO
}

\author{
R. Neuhäuser ${ }^{1} \dagger$, A. Seifahrt ${ }^{1,2}$, T. Röll ${ }^{1}$, \\ A. Bedalov ${ }^{1}$, and M. Mugrauer ${ }^{1}$ \\ ${ }^{1}$ Astrophysikalisches Institut, Universität Jena, Schillergäßchen 2-3, 07745 Jena, Germany \\ ${ }^{2}$ European Southern Observatory, Karl-Schwarzschild-Str. 2, 85748 Garching, Germany
}

\begin{abstract}
Many planet candidates have been detected by radial-velocity variations of the primary star; they are planet candidates, because of the unknown orbit inclination. Detection of the wobble in the two other dimensions, to be measured by astrometry, would yield the inclination and, hence, true mass of the companions. We aim to show that planets can be confirmed or discovered in a close visual stellar binary system by measuring the astrometric wobble of the exoplanet host star as a periodic variation of the separation, even from the ground. We test the feasibility with HD 19994, a visual binary with one radial velocity planet candidate. We use the adaptive optics camera NACO at the VLT with its smallest pixel scale $(\sim 13$ mas $)$ for high-precision astrometric measurements. The separations measured in 120 single images taken within one night are shown to follow white noise, so that the standard deviation can be divided by the square root of the number of images to obtain the precision. In this paper we present the first results and investigate the achievable precision in relative astrometry with adaptive optics. With careful data reduction it is possible to achieve a relative astrometric precision as low as 50 $\mu$ as for a $0^{\prime \prime} .6$ binary with VLT/NACO observations in one hour, the best relative astrometric precision ever achieved with a single telescope from the ground. The relative astrometric precision demonstrated here with $\mathrm{AO}$ at an 8-m mirror is sufficient to detect the astrometric signal of the planet HD $19994 \mathrm{Ab}$ as periodic variation of the separation between HD $19994 \mathrm{~A}$ and B.
\end{abstract}

\section{Introduction}

Since the radial velocity technique can yield only lower mass limits, all planets (or planet candidates) found by this technique have to be confirmed by other methods. Of $\sim 200$ radial velocity planet candidates found so far, 14 have been confirmed by transit and two by astrometry (see, e.g., exoplanet.eu.) The two planets GJ 876b and 55 Cancri d have been confirmed by astrometry using the Hubble Space Telescope Fine Guidance Sensor (Benedict et al. 2002, McArthur et al. 2004), with a precision down to 0.04 mas (milliarcsec), sufficient to detect the wobble of the host star in the plane of the sky.

The astrometric displacement is given by

$$
\theta[\mathrm{mas}]=0.960 \times \frac{a}{[5 \mathrm{AU}]} \times \frac{[10 \mathrm{pc}]}{d} \times \frac{M_{\mathrm{pl}}}{\left[M_{\mathrm{jup}}\right]} \times \frac{\left[M_{\odot}\right]}{M_{\star}}
$$

with planet mass $M_{\mathrm{pl}}$ in Jupiter masses at a semi-major axis a (in units of $5 \mathrm{AU}$ ) in a circular orbit around a host star with mass $M_{\star}$ in solar masses at a distance $d$ (in units of $10 \mathrm{pc})$.

Here, we present both a feasibility study to determine the astrometric precision of the Adaptive Optics (AO) camera NAos-COnica (NACO) of the ESO Very Large Telescope

\section{† rne@astro.uni-jena.de}




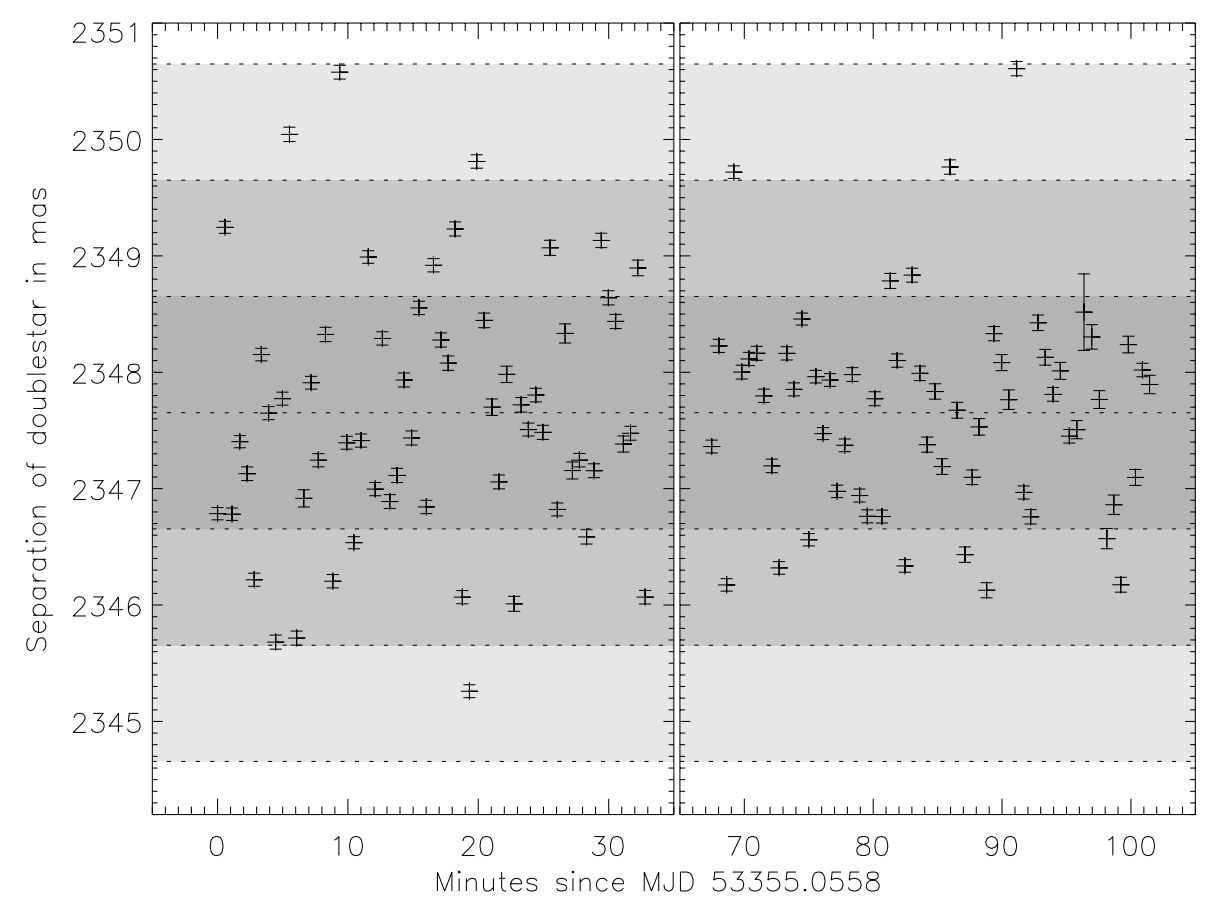

Figure 1. Measured separation for HD 19994 (similar for HD 19063). Error bars denote $1 \sigma$ error for individual measurements. The standard deviation of the complete dataset is shown as dashed lines and grey-shaded areas for 1,2 , and $3 \sigma$, respectively. The standard deviation of the mean, thus the precision of the measured binary separation, is $0.998 / \sqrt{120}$ mas $=91.2 \mu$ as .

(VLT) as well as first measurements in order to determine the mass of HD $19994 \mathrm{Ab}$, a RV planet candidate in orbit around HD 19994: The RV data suggest a lower mass limit of the planet candidate of $m \cdot \sin i=1.68 \mathrm{M}_{\mathrm{Jup}}$ in an orbit with semi-major axis $a=1.42$ AU with an eccentricity of $e=0.30 \pm 0.04$, i.e., a $535.7 \pm 3.1$ day orbital period (Mayor et al. 2004). The full (peak-to-peak) astrometric wobble, from Kepler's $3^{\text {rd }}$ Law, is at least 0.155 mas (for a circular orbit) and 0.131 mas by taking the observed eccentricity into account - this is assuming the minimum mass $m \cdot \sin i$ as the true mass.

\section{Observations and data reduction}

We observed HD 19994 and a calibration binary (HD 19063) with the S13 camera $\left(13.26 \mathrm{mas} /\right.$ pixel pixel scale, $\left.14^{\prime \prime} \times 14^{\prime \prime} \mathrm{FOV}\right)$ in a NB_2.17 narrow-band filter. Short exposures of both target binaries were taken for one hour each, equally split up into two time slots before and after meridian passage. We obtained a total of 120 frames for HD 19994 and 117 frames for HD 19063. Data reduction followed the standard technique of dark subtraction, division by a flat field and the application of a bad pixel mask with the eclipse software. In each single exposure $(0.347 \mathrm{sec})$, we measured the separation between $\mathrm{A}$ and $\mathrm{B}$ of both binaries. We have then made sure that the separations follow white noise by a K-S test (see a future paper by Neuhäuser et al. for details). Hence, we can divide the standard deviation of the mean by the square root of the number of measurements to obtain the precision of the separation measurement, the precision, not the accuracy. For the latter, we would need to know the true pixel scale. See Figure 1 and Table 1. 
Table 1. Astrometric results from the measurements of the binaries HD 19994 and HD 19063. K-S test values higher than 0.6 are interpreted as indicating white noise.

\begin{tabular}{cccccr}
\hline Target & $\begin{array}{c}\text { Mean sepa- } \\
\text { ration (mas) }\end{array}$ & $\begin{array}{l}\text { Standard } \\
\text { deviation }\end{array}$ & N & $\begin{array}{r}\text { K-S } \\
\text { test }\end{array}$ & $\begin{array}{r}\text { Precision } \\
\text { achieved }\end{array}$ \\
\hline HD 19994 & 2347.652 & 0.998 mas & 120 & 0.69 & $91.2 \mu \mathrm{as}$ \\
& 2347.632 & 1.110 mas & 60 & 0.85 & $143.3 \mu \mathrm{as}$ \\
HD 19063 & 2347.673 & 0.882 mas & 60 & 0.64 & $113.9 \mu \mathrm{as}$ \\
& 649.628 & 0.377 mas & 57 & 0.74 & $49.9 \mu \mathrm{as}$ \\
& 649.596 & 0.391 mas & 60 & 0.48 & (failed) \\
\hline
\end{tabular}

Differential chromatic refraction (DRC) or Allan noise (see Pravdo \& Shaklan 1996) do not matter here, because we observe in the near IR with a narrow-band filter (hence no DCR) and with AO (hence no Allan noise).

\section{Results and conclusions}

We have observed two bright visual binaries (0.6 to 2.4 arc sec separation) with VLT/NACOs smallest pixel scale (13.26 mas/pixel) by taking $\sim 120$ short $(0.347 \mathrm{~s})$ exposures per binary within a few hours, separated in four bins of $\sim 60$ exposures each (15 December 2004, ESO program 075.C-0288.A). We could confirm that the separations measured follow white noise (in three out of four bins), so that we are allowed to divide the mean of the separations by the square root of the number of measurements to obtain the precision of the separation measurement. By doing so, we measured the separation between HD $19994 \mathrm{~A}$ and B to be 2347.652 mas (milliarcseconds) with the relative precision of $91.2 \mu \mathrm{as}$ (microarcseconds). For HD 19063, we obtained 649.628 mas with a precision being as low as $49.9 \mu a s$. These are the most precise measurements in relative astrometry obtained with single-aperture telescopes from the ground, sufficient to detect the astrometric wobble due to a planet, to be seen as periodic change in the binary separation.

Such a high precision as shown here can be applied not only to measurements of masses of previously-detected RV planets (or candidates), but also to search for new planets in wide binaries, or even single stars with a bright (back- or foreground) star within the isoplanatic angle. Other possible applications are orbit determinations in stellar multiples, ground-based parallax and proper motion measurements, observations of expanding, contracting, or rotating star clusters, etc.

The main limitation of this technique is the need for a bright star near the target, namely within the isoplanatic angle. And, as long as no pixel scale calibrator is available with a precision down to $1 / 100000$, the measurement can achieve this high precision only for relative astrometry, but not in absolute terms.

\section{References}

Benedict, G.F., McArthur, B.E., Forveille, T., et al., 2002, ApJ 581, L115

Mayor, M., Udry, S., Naef, D., Pepe, F., Queloz, D., Santos, N.C., \& Burnet, M. 2004, A\&A 415,391

McArthur B.E., Endl M., Cochran W.D., et al., 2004, ApJ 614, L81

Pravdo, S.H., \& Shaklan, S.B. 1996, ApJ 465, 264 